\title{
Thin Endometrium and Cleavage Embryo Transfer Are Associated With Ectopic Pregnancy After in Vitro Fertilization-embryo Transfer Cycles: a Matched Case-control Study Using Propensity Score Matching
}

\section{Ying Zhao}

Reproductive Medicine Center, Xiangya Hospital, Central South University

Dong'e Liu

Reproductive Medicine Center, Xiangya Hospital, Central South University

Nenghui Liu

Reproductive Medicine Center: University of Minnesota Physicians

\section{Yumei Li}

Reproductive Medicine Center, Xiangya Hospital, Central South University

\section{Zhongyuan Yao}

Reproductive Medicine Center: University of Minnesota Physicians

\section{Fen Tian}

Reproductive Medicine Center, Xiangya Hospital, Central South University

Aizhuang $\mathbf{X u}$

Reproductive Medicine Center, Xiangya Hospital, Central South University

Yanping Li ( $\square$ liyanp@csu.edu.cn )

Reproductive Medicine Center, Xiangya Hospital, Central South University https://orcid.org/0000-00024193-7321

\section{Research}

Keywords: Ectopic pregnancy, EMT, Endometrial pattern, Endometrial growth amplitude, In vitro fertilization, Risk factors, Cleavage stage embryo, Blastocyst

Posted Date: February 19th, 2021

DOl: https://doi.org/10.21203/rs.3.rs-214690/v1

License: (a) This work is licensed under a Creative Commons Attribution 4.0 International License. Read Full License 


\section{Abstract}

Objective: To evaluate the relationship between the endometrial features (endometrial thickness(EMT), pattern and endometrium growth amplitude and rate) and the embryo stage transferred and ectopic pregnancy after IVF-ET based on the data of 628 matched cases.

Methods: This was a 1:1 matched case-control study that enrolled 314 EP patients and 314 matched IUP patients from the ART center of Xiangya Hospital of Central South University from January 2014 to April 2020. Univariate analysis and multiple-stepwise logistic regression analysis was used to analyze the risk factors of EP, and a receiver-operating characteristic (ROC) curve was generated to predict EP.

Results: 1. The EP group had a higher cleavage stage embryo transfer rate( $94.57 \%$ vs $86.22 \%)$, a thinner endometrium on transformation day(the day when progesterone was added in the frozen embryo cycle or the trigger day in the fresh embryo cycle) $(9.40(2.60) \mathrm{mm}$ vs $9.80(2.60) \mathrm{mm})$ compared with IUP group. In fresh embryo cycles, the EP group had a higher endometrium pattern $\mathrm{C}$ proportion on transformation day, a smaller endometrium increment, and a slower endometrial growth rate compared with the IUP group.

2.The ROC curves were used to analyze the cut-off values of the EMT on transformation day, the endometrial growth amplitude and rate in fresh cycles, the results were $9.35 \mathrm{~mm}, 4.90 \mathrm{~mm}$ and 0.491 $\mathrm{mm} / \mathrm{d}$, respectively. The incidence of EP was significantly different between groups according to cut-off values. 3.The transferred embryo stage and EMT on transformation day were independent factors affecting outcome in the general population. The area under the curve(AUC) of the EMT and the stage of embryos transferred for EP prediction was 0.604 , sensitivity was 0.702 , and specificity was 0.465 . The EMT on trigger day was an independent factor affecting outcome in the fresh cycles. The AUC of the EMT for EP prediction in fresh cycles was 0.605 , sensitivity was 0.805 , and specificity was 0.420 .

Conclusions: 1. Transferring cleavage stage embryo, and thin EMT on the transformation day were risk factors for EP. 2. Thin EMT and pattern $\mathrm{C}$ on transformation day may be related to abnormal endometrial receptivity and endometrial peristaltic waves. 3.The combined indicator of EMT and embryonic development stage could not well predict the occurrence of EP after ET.

\section{Background}

Ectopic pregnancy (EP) is a kind of pregnancy in which the embryo implants outside the uterine cavity [1]. It is reported that EP deaths account for $5 \%$ and $10 \%$ of all maternal deaths in developed and developing countries, respectively ${ }^{[2]}$.

The risk factors of EP include fallopian tubes factors ${ }^{[3-8]}$, older maternal age ${ }^{[9,10]}$, infertility history $[4]$, endometriosis ${ }^{[4,11,12]}$ and assisted reproductive technology treatment ${ }^{[3]}$. In theory, ART treatment can reduce the risk of ectopic pregnancy, because neither fertilization nor embryo transfer involves the fallopian tubes. 
In fact, the first pregnancy achieved by IVF was ectopic ${ }^{[13]}, 2$ years before the birth of the worldwide first IVF-baby reported ${ }^{[14]}$. The ectopic pregnancy rate is about $1-2 \%$ in spontaneous pregnancy and as high as $1.0-11 \%$ in $A R T^{[15,16]}$. ART plays an important role in the occurrence of ectopic pregnancy ${ }^{[17]}$. However, data on risk factors for EP after IVF were inconsistent. Risk factors associated with ectopic pregnancy after ART include tubal infertility ${ }^{[5,18-22]}$, multiple embryos transferred ${ }^{[18]}$, and fresh embryo transfer compared with thawed embryo transfer cycles in stimulated cycles ${ }^{[23-28]}$. It is controversial whether the development stage of the embryos transferred affects the incidence of EP ${ }^{[29-32]}$.

Few studies were focus on the correlation and predictive ability of combined endometrial and embryotic features to ectopic pregnancy after ART treatments, and there is no consensus on the cut-off value of EMT associated with ectopic pregnancy.

Thus, it is important to identify risk factors for EP, and best to find out the relevant combined endometrial and embryonal indicators that can predict EP related to ART, so as to guide embryo transfer to a certain extent. Considering the relatively low incidence rate of EP, and many factors are associated with EP, we designed a matched case-control study using propensity score matching to assess the relationship between endometrial features (endometrial pattern, thickness and endometrium growth amplitude and daily rate) and stage of embryo transferred and EP based on 628 in vitro fertilization-embryo transfer cycles.

\section{Methods}

\section{Ethical approval}

The present study was approved by the Ethics Committee of Xiangya Hospital, Central South University. For patients who receive ART (IVF/ICSI) treatment in our center, their medical records are allowed to be used for research. All patients received informed consent before ART treatment. All patients were registered in data management system, which is used to store all medical information about patients trying to conceive through ART.

\section{Definition of clinical outcomes}

When one or more gestational sacs that were confirmed in the uterus by ultrasonic examination, intrauterine pregnancy was defined. The definition of ectopic pregnancy was the observation of one or more gestational sacs outside the uterine cavity under ultrasound. Heterotopic pregnancy was defined as the coexistence of pregnancy inside and outside the uterine cavity. According to the ART follow-up flow path in our center, all patients were asked to come back for a blood $\beta$-human chorionic gonadotropin ( $\beta$ hCG) test 12 days after ET. $\beta$-hCG levels below 5 IU/L were considered negative, those between 5 and 15 $\mathrm{IU} / \mathrm{L}$ were considered indeterminate, and those above $15 \mathrm{IU} / \mathrm{L}$ were considered positive. Patients with indeterminate $\beta$-hCG levels were considered positive if their $\beta$-hCG increased after $48 \mathrm{~h}$. Those patients with increasing $\beta$-hCG were followed sonographically 28 days after ET for the first time, especially for 
those with a history of EP or previous tubectomy to rule out EP earlier. In addition, 35 days after ET, they underwent a second ultrasound scan to confirm the presence of a gestational sac with fetal heart in the uterus (especially transvaginal). Only a small number of the diagnoses of ectopic/heterotopic pregnancy were made in other hospitals; for those cases, we confirmed that the diagnostic criteria were the same as those in our hospital. As the main purpose of our study was to investigate endometrial and embryo factors associated with the occurrence of EP, heterotopic pregnancy was excluded in our analysis.

\section{Risk factor selection}

We used the female age ${ }^{[9,10,33]}$, body mass index $(\mathrm{BMI})^{[34]}$, the duration of infertility, gravidity ${ }^{[35]}$, previous history of ectopic pregnancy $\left.{ }^{[36,37}\right]$, ovarian stimulation/endometrial preparation protocol ${ }^{[38]}$, number of embryos transferred ${ }^{[18,35]}$, the main causes of infertility ${ }^{[18]}$, and frozen-thawed/ fresh embryos transferred ${ }^{[39,40]}$ as matching factors because previous study have determined that these factors are associated with the occurrence of EP, and our goal in the present study was to analyze the relationship between endometrial features and embryonic development stage and EP. Our study reduced bias of population selection by adjustment of matching the time for infertility treatment. The sample sizes were 314 and 314 in the EP and IUP groups with a ratio of 1:1, based on the propensity score with a standard caliper width of 0.2 .

\section{Study design and patients}

This was a 1:1 case-control study of all patients who received IVF treatment between January 2014 and April 2020 at the Center for Assisted Reproductive Technology, Xiangya Hospital, Central South University, China. Data were extracted from clinical records. The selection process for IVF cycles is illustrated in Fig. 1. From the initial 21704 patients, we selected 11699 patients with a positive hCG value (12 days after embryo transfer). Patients were eligible if they met the following criteria: (1) good physical and mental health (not disabled and without psychological disorders and mental illness); (2) IVF/intracytoplasmic sperm injection (ICSI) cycle; and (3) positive hCG test 12 days after an embryo transfer. The exclusion criteria were as follows: (1) biochemical pregnancy; (2) cornual pregnancy; (3) cesarean scar pregnancy; (4) cervical pregnancy and (5) heterotopic pregnancy. We further selected patients resulted in a clinical pregnancy of either EP or IUP. Endometrial thickness, growth and pattern were assessed at two time points (the day of gonadotrophin stimulation and HCG administration in fresh embryo cycle, the first day use estrogen and the day of progesterone addition in the hormone treatment frozen embryo cycle, or the 10th day of menstruation and the day of ovulation of frozen embryos in the ovulation cycle), the increment and growth rate of endometrium within cycle were calculated simultaneously. The EMT was obtained in a sagittal midline plane on transvaginal sonography, with the maximal anteroposterior thickness used, in the method described by Bredella et al ${ }^{[41]}$. The echoes of the endometrium was observed, the endometrial patterns were divided into three types based on the Gonen system ${ }^{[42]}$ : type A, trilaminar pattern (endometrial three-layer pattern), consisting of hyperechoic outer and middle layers, hypoechoic inner layers, and evident echo at the intrauterine midline; type $B$, relatively homogeneous hyperechoic endometrium, with unclear endometrial layers, obscure intrauterine midline echo, but clear 
interface between endometrial and muscular layers; and type $C$, homogeneous hyperechoic endometrium without intrauterine midline echo. The developmental stage of the embryos transferred were recorded at the same time.

\section{Statistics}

All statistical analyses were carried out using R 3.6.2 statistical software. For the quantitative data, the mean \pm SD and median (quartile interval) are used to describe the normal distributed data and nonnormal distributed data respectively. For categorical data, the number of cases (percentage) is used to describe it.

In the comparison of univariate differences, two independent samples t-test was used for those who is normally distributed, Mann-Whitney $U$ test for those who was not in the normal distribution, and Pearson Chi-square test was used for the categorical variables. Fisher's exact probability method was used when $\mathrm{n}$ $<40$ or $\mathrm{T}<1$ in the Chi-square test table( $\mathrm{n}$ is the total number of samples, $\mathrm{T}$ is the theoretical frequency). Stepwise multiple logistic regression analysis was used to analyze the risk factors for EP, and a receiveroperating characteristic(ROC) curve was generated for the predictors of EP. The validity of the model was assessed by AUC. In this research, $\mathrm{P}<0.05$ was considered statistically significant difference.

\section{Results}

A total of 314 ectopic pregnancies and 314 matched intrauterine pregnancies were included in this study. The baseline characteristics of the two groups are shown in Table 1. There were no significant differences in the female ages, body mass index (BMI), infertility duration, number of embryos transferred, times of previous pregnancies and ectopic pregnancies, fresh/frozen embryo, protocol and main cause of infertility between the two groups. 7po

Table 1 The baseline characteristics of the patients in the two groups 


\begin{tabular}{|c|c|c|c|}
\hline & IUP $(n=314)$ & $\operatorname{EP}(n=314)$ & $P$ \\
\hline Female age & $30.00(6.00)$ & $30.00(6.00)$ & 0.309 \\
\hline BMI & $21.50(4.26)$ & $21.09(3.74)$ & 0.178 \\
\hline Infertility duration & $4.00(4.00)$ & $4.00(4.00)$ & 0.617 \\
\hline Number of embryos transferred & $2.00(0.00)$ & $2.00(0.00)$ & 0.707 \\
\hline Gravidity & & & 0.694 \\
\hline 0 & $141(44.90)$ & 133(42.36) & \\
\hline 1 & $59(18.79)$ & $70(22.29)$ & \\
\hline 2 & $68(21.66)$ & $63(20.06)$ & \\
\hline 3 & $23(7.32)$ & $26(8.28)$ & \\
\hline 4 & $11(3.50)$ & $15(4.78)$ & \\
\hline 5 & $5(1.59)$ & $4(1.27)$ & \\
\hline 6 & $7(2.23)$ & $3(0.96)$ & \\
\hline Times of previous ectopic pregnancies & & & 0.977 \\
\hline 0 & 243(77.39) & 239(76.11) & \\
\hline 1 & $47(14.97)$ & $49(15.61)$ & \\
\hline 2 & $21(6.69)$ & $23(7.32)$ & \\
\hline 3 & $3(0.96)$ & $3(0.96)$ & \\
\hline Cycle & & & 0.805 \\
\hline Frozen-thawed embryo transfer & $172(54.78)$ & $165(52.55)$ & \\
\hline Fresh embryo transfer & $142(45.22)$ & $149(47.45)$ & \\
\hline Protocol & & & 0.539 \\
\hline Hormone treatment frozen embryo cycle & $113(35.99)$ & $106(33.76)$ & \\
\hline Frozen embryo cycle after ovulation & $60(19.11)$ & $57(18.15)$ & \\
\hline Antagonist & $10(3.18)$ & $18(5.73)$ & \\
\hline Agonist & $93(29.62)$ & $89(28.34)$ & \\
\hline Others & $38(12.10)$ & $44(14.01)$ & \\
\hline Main course of infertility & & & 0.060 \\
\hline Sequelae stage of pelvic inflammatory disease & $301(95.86)$ & $307(97.77)$ & \\
\hline
\end{tabular}




\begin{tabular}{|lcc|} 
Endometriosis & $0(0.00)$ & $2(0.64)$ \\
\hline Others & $13(4.14)$ & $5(1.59)$ \\
\hline
\end{tabular}

$E P$ ectopic pregnancy, IUP intrauterine pregnancy, $B M /$ body mass index

Compared with the IUP group, EP women had a significantly higher cleavage stage embryo transfer rate $(P<0.001)$, significantly thinner EMT on transformation day $(P<0.001)($ Table 2$)$.

For fresh cycles, EP women had a significant higher ratio of endometrial pattern $\mathrm{C}$ on transformation day (10.96\% v 4.29\%, $P=0.047)$, smaller endometrial growth amplitude $(P=0.009)$, and slower endometrial daily growth rate $(P=0.022)$ compared with IUP women. There were no difference in the above indicators for women who transferred frozen embryos(Table 2).

EMT and pattern at the first time point(the day of gonadotrophin stimulation in fresh embryo cycle, the first day using estrogen in the hormone treatment frozen embryo cycle, or the 10th day of menstruation of frozen embryos in the ovulation cycle) in the two groups were similar either by integral analysis or analyzed in subgroups according to the fresh/frozen embryo cycle (Table 2).

Table 2 Comparison of endometrial and embryotic parameters in EP and IUP women 


\begin{tabular}{|c|c|c|c|}
\hline & $\operatorname{EP}(n=314)$ & $\begin{array}{l}\text { IUP } \\
(n=314)\end{array}$ & $P$ \\
\hline The stage of embryos transferred & & & $<0.001 *$ \\
\hline cleavage stage embryo & $269(86.22)$ & 296(94.57) & \\
\hline blastocyst & $43(13.78)$ & $17(5.43)$ & \\
\hline EMT on the first point & $5.30(2.70)$ & $5.30(2.85)$ & 0.913 \\
\hline EMT on the first point (Fresh embryo cycles) & $4.70(1.98)$ & $4.90(2.23)$ & 0.468 \\
\hline $\begin{array}{l}\text { EMT on the first point (Hormone treatment frozen embryo } \\
\text { cycle) }\end{array}$ & $5.40(3.33)$ & $5.30(3.20)$ & 0.600 \\
\hline EMT on the first point (Frozen embryo cycle after ovulation) & $6.60(2.40)$ & $6.20(2.70)$ & 0.136 \\
\hline Endometrial pattern on the first point & & & 0.990 \\
\hline A & $18(6.45)$ & $18(6.64)$ & \\
\hline B & $93(33.33)$ & $89(32.84)$ & \\
\hline C & 168(60.22) & $164(60.52)$ & \\
\hline Endometrial pattern on the first point (Fresh embryo cycles) & & & 0.472 \\
\hline A & $0(0.00)$ & $2(1.71)$ & \\
\hline B & $26(22.03)$ & $23(19.66)$ & \\
\hline C & $92(77.97)$ & $92(78.63)$ & \\
\hline $\begin{array}{l}\text { Endometrial pattern on the first point (Hormone treatment } \\
\text { frozen embryo cycle) }\end{array}$ & & & 0.533 \\
\hline A & $10(10.10)$ & $7(7.45)$ & \\
\hline B & $25(25.25)$ & $30(31.91)$ & \\
\hline C & $64(64.65)$ & $57(60.64)$ & \\
\hline $\begin{array}{l}\text { Endometrial pattern on the first point (Frozen embryo cycle } \\
\text { after ovulation) }\end{array}$ & & & 0.663 \\
\hline A & $8(12.90)$ & $9(15.00)$ & \\
\hline B & $42(67.74)$ & $36(60.00)$ & \\
\hline C & 12(19.35) & $15(25.00)$ & \\
\hline EMT on transformation day & $9.80(2.60)$ & $9.40(2.60)$ & $0.004^{*}$ \\
\hline EMT on transformation day (Fresh embryo cycles) & $10.90(2.60)$ & $9.80(2.90)$ & $0.002^{*}$ \\
\hline $\begin{array}{l}\text { EMT on transformation day (Hormone treatment frozen } \\
\text { embryo cycle) }\end{array}$ & $9.30(1.80)$ & $9.05(1.90)$ & 0.468 \\
\hline
\end{tabular}


EMT on transformation day (Frozen embryo cycle after ovulation)

$9.70(2.15) \quad 9.30(2.10) \quad 0.384$

Endometrial pattern on transformation day

0.311

$\begin{array}{lcc}\text { A } & 123(39.30) & 109(35.05) \\ \text { B } & 172(54.95) & 176(56.59) \\ \text { C } & 18(5.75) & 26(8.36)\end{array}$

Endometrial pattern on transformation day (Fresh embryo cycles)
A
62(44.29) 50(34.25)
B
72(51.43) $\quad 80(54.79)$
C
$6(4.29)$
$16(10.96)$

Endometrial pattern on transformation day (Hormone treatment frozen embryo cycle)
A
$31(28.18) \quad 30(28.85)$
B
72(65.45) 69(66.35)
C
$7(6.36)$
$5(4.81)$

Endometrial pattern on transformation day (Frozen embryo cycle after ovulation)

\begin{tabular}{|c|c|c|c|}
\hline A & $30(47.62)$ & $29(47.54)$ & \\
\hline $\mathrm{B}$ & $28(44.44)$ & $27(44.26)$ & \\
\hline C & $5(7.94)$ & $5(8.20)$ & \\
\hline Endometrial growth amplitude & $4.30(3.70)$ & $3.90(3.23)$ & 0.082 \\
\hline Endometrial growth amplitude (Fresh embryo cycles) & $6.05(2.70)$ & $4.90(4.05)$ & $0.009 *$ \\
\hline $\begin{array}{l}\text { Endometrial growth amplitude (Hormone treatment frozen } \\
\text { embryo cycle) }\end{array}$ & $3.90(2.92)$ & $3.35(3.22)$ & 0.481 \\
\hline $\begin{array}{l}\text { Endometrial growth amplitude (Frozen embryo cycle after } \\
\text { ovulation) }\end{array}$ & $2.91 \pm 1.97$ & $3.15 \pm 1.82$ & 0.480 \\
\hline Endometrial daily growth rate & $0.47(0.38)$ & $0.43(0.40)$ & 0.173 \\
\hline Endometrial daily growth rate (Fresh embryo cycles) & $0.56(0.32)$ & $0.47(0.41)$ & $0.022^{*}$ \\
\hline $\begin{array}{l}\text { Endometrial daily growth rate (Hormone treatment frozen } \\
\text { embryo cycle) }\end{array}$ & $0.36(0.25)$ & $0.32(0.30)$ & 0.474 \\
\hline $\begin{array}{l}\text { Endometrial daily growth rate (Frozen embryo cycle after } \\
\text { ovulation) }\end{array}$ & $0.50(0.46)$ & $0.57(0.40)$ & 0.532 \\
\hline
\end{tabular}




\section{* $P<0.05$}

For the quantitative data, the mean $\pm \mathrm{SD}$ and median (quartile interval) are used to describe the normal distributed data and non-normal distributed data respectively. For categorical data, the number of cases (percentage) is used to describe it.

Using the ROC curve to generate cut-off values for the significant indicators above, the results for EMT on trigger day, endometrial growth amplitude, and daily growth rate in fresh cycles were $9.35 \mathrm{~mm}, 4.90 \mathrm{~mm}$ and $0.491 \mathrm{~mm} / \mathrm{d}$, respectively(Fig. 2,3,4).

Stepwise logistic regression analysis was performed to further investigate the risk factors for EP. The regression model selected the variables which were significant in univariate analysis. Finally, cleavage embryo transfer and a thin EMT on transformation day were independent risk factors for developing EP following ART[Table 3]. Stepwise logistic regression analysis was also performed in subgroups. For fresh cycles, a thin EMT on trigger day was an independent risk factor for developing EP[Table 4].

Table 3 Stepwise logistic regression analysis for the general population

\begin{tabular}{|lll|}
\hline & OR(95Cl\%) & $P$ \\
\hline $\begin{array}{l}\text { The stage of embryos transferred } \\
\text { cleavage stage embryo }\end{array}$ & 1.00 & 0.000 \\
\hline blastocyst & $0.334(0.180,0.593)$ & \\
\hline EMT on the transformation day & $0.868(0.799,0.942)$ & 0.001 \\
\hline
\end{tabular}

EP is more likely to occur in patients who transferred cleavage embryos or had a thinner EMT on the transformation day.

Table 4 Stepwise logistic regression analysis for fresh cycles

\begin{tabular}{|lll|}
\hline & OR(95Cl\%) & $\boldsymbol{P}$ \\
\hline EMT on transformation day & $0.842(0.742,0.95)$ & 0.0063 \\
\hline
\end{tabular}

In fresh cycles, EP is more likely to occur in patients had a thinner EMT on the transformation day.

As showed in Fig. 5, a prediction model was generated based on EMT on transformation day and the embryo stage for ectopic pregnancy, ROC curve was used to assess the ability of the model. The area under the curve which calculated as sensitivity/(1-specificity), is a measure that can show 'superior' (AUC close to 1) or 'inferior' (AUC close to 0.5 ) the model is. In the present study, the results from ROC curve analysis suggest these endometrial and embryo parameters have poor predictive value for ectopic pregnancy(AUC $0.604,95 \% \mathrm{Cl} 0.64-0.72$ ); The subgroup analysis of fresh embryo cycles based on the endometrium thickness on trigger day showed similar result s (AUC 0.605, 95\% Cl 0.64-0.72)(Fig. 6). 


\section{Discussion}

With the hope to provide suggestions for EP prevention, this matched case-control study was designed to identify risk factors related to EP in women undergoing ART working backwards from the outcome to exposure. To our knowledge, this is the first study to analyze only endometrial and embryonic developmental factors associated with ectopic pregnancy after precise matching of other risk factors.

In this study, transferring cleavage stage embryo was found to be a risk factor of EP. This is consistent with some previous studies, which have shown that blastocyst transfer may reduce EP in IVF/intracytoplasmic sperm injection (ICSI) cycles ${ }^{[39,43,44]}$, and day-3 embryo transfers was an ectopic pregnancy risk factor in IVF ${ }^{[45]}$. In the case of cleavage-stage ETs, the embryos are not temporally prepared for immediate implantation regardless of whether the endometrium is in a receptive state and can migrate within the upper female reproductive tract before implantation. Selecting blastocysts has the advantage of physiological synchronization with the uterine endometrium, therefore, it may lead to better pregnancy outcomes ${ }^{[46]}$. Meanwhile, it has been reported that uterine contractile decreased on day 5 , which may be another reason for the decreased EP rate after blastocyst transfer ${ }^{[30]}$. But in contrast, many studies ${ }^{[30,47-50]}$ considered the stage of embryos did not affect the EP rate. In addition, other researches have indicated that blastocyst transfer may increase the risk of EP compared with the cleavage stage embryo transfer on account of the potentially higher implantation rate of each blastocyst ${ }^{[31,51]}$. The reason why they did not come to the same conclusion may be related to different research sample sizes, patients' age, experimental designs and analysis methods among studies, even blastocyst culture techniques in reproductive centers. In our center, almost all embryos transferred in the fresh cycle are cleavage stage, blastocysts can only be transferred in the frozen cycle, which leads to the lack of data of blastocyst transfer in the fresh cycle, which may be one of the reasons why our results are different from some previous studies. Although EP risk was lower when the blastocyst was transferred, all-blastocystincubation strategy is likely to lead to zero blastocyst formation and canceling of transfer for the elderly women, this will be a huge blow to the elderly couples hoping to have a baby.

The success of in vitro fertilization and embryo transfer (IVF-ET) cycles depends primarily on embryo quality and uterine receptivity, both are indispensable. It's speculated that mismatch between the embryo stage transferred and the receptive state of the endometrium can lead to increased incidence of EP after ART treatment, as it will lead to inadequate embryonal-endometrial crosstalk, the blastocyst travels in the uterine cavity for a longer time to wait for the endometrial implantation window, it is also possible that the endometrium is not capable of receiving embryo implantation, increasing the likelihood that the embryo migrating outside the uterus, eventually leading to an EP.

A variety of studies have suggested that ultrasound can be used as a noninvasive and simple method to assess the endometrial receptivity ${ }^{[52]}$. Several sonographic parameters have been evaluated, including EMT, endometrial pattern, endometrial volume and endometrial and subendometrial blood flow ${ }^{[53-57]}$. Many studies have proposed that a correlation exists between EMT and uterine receptivity ${ }^{[58-63]}$. Few 
studies use EMT during ART therapy to predict future EP, the cutoff value for EP associated EMT is also debated. One study showed that the EMT > $12 \mathrm{~mm}(\mathrm{OR} 0.27 ; 95 \% \mathrm{Cl} 0.13-0.56)$ prior to embryo transfer was a protective factor against $E P^{[64]}$. Our study showed that, for fresh cycles, the EMT of the EP group on transformation day was significantly lower than that of the IUP group, the best cutoff point was $9.35 \mathrm{~mm}$, the thinner the endometrial was, the more likely EP would occur. However, for frozen cycles, we came to the opposite conclusion from Hongfang Liu's study ${ }^{[65]}$, no significant difference was found in EMT between the two groups.

It's known the endometrium can be divided into basal layer and functional layer. The functional layer is a highly dynamic tissue that changes periodically under the action of steroid hormones, creating an endometrial implantation window ${ }^{[66]}$. The ultrasonic appearance of the endometrium reflects these periodic changes. It can be considered that the endometrium measured on the first time point was the basal layer, and endometrial growth amplitude represents the functional layer thickness at the late proliferative stage (EMT on transformation day minus EMT on the first time point). Take into account the different length of stimulation in each patient, we included the average daily growth rate of the endometrium in our analysis. The results showed that there was no difference in EMT on the first time point between the two groups, suggesting that the thickness of the basal layer is always constant in most patients. In fresh cycles, the endometrial growth amplitude and daily growth rate of EP patients were significantly smaller/slower than that of IUP group, the cutoff value were $4.9 \mathrm{~mm}$ and $0.491 \mathrm{~mm} / \mathrm{d}$, respectively. However, for the frozen embryo cycles, these indicators had no difference between two groups. In general, all the studies showed that thinner EMT may be associated with worse endometrial receptivity in fresh cycles. A previous study confirmed that an increased EMT was positively correlated with an increased risk of placenta praevia ${ }^{[67]}$, hypothesized that increased EMT is a sign of the frequency and/or amplitude of uterine peristalsis wave, which may increase the risk of the embryos being dislodged from their initial transfer location. Combined with our results, it can be speculated that EMT may be related to the direction of endometrial peristaltic waves, thicker endometrium may represent fundus-tocervix uterine peristalsis, leading to a higher incidence of placenta praevia [67], as well as a lower EP rate in the present. In frozen embryos cycle, endometrium growth depends on exogenous estrogen stimulation, rather than the hyperphysiological levels of endogenous estrogen caused by multiple follicles develop simultaneously, it can be assumed that only very high levels of hormones affect the direction of the endometrial peristaltic waves. A prospective study to determine the actual endometrial implant window by combining molecular biology with pregnancy outcome tracking and to measure the endometrial peristaltic wave, and to analyze their association with different endometrial parameters should be interesting. Further larger studies should confirm a more accurate cutoff value, to guide clinical embryo transfer so as to avoid the occurrence of EP as much as possible.

Our study indicated that in fresh cycles, when endometrial pattern on transformation day was C, EP was more likely to occur. It has been reported that women with lower implantation rates and pregnancy rates show homogenous patterns, although there are conflicting results ${ }^{[68-70]}$. It is generally believed that the hyperechoic middle line represents the uterine cavity, and the other two hyperechogenic line are related to 
the endometrium-myometrium interface, but the main reason for the hyperechoic structure of secretory endometrium is still controversial. Fleischer et al. ${ }^{[71]}$ suggested that the homogeneous hyperechoic endometrium during the late secretory phase might indicate the stromal edema by comparing the endometrial chronological date with glandular histology and stromal histology respectively ${ }^{[72]}$. The transformation process of endometrial from proliferative phase to secretory phase under the influence of hormones is called endometrial decidualization. The disability of decidualization is related to recurrent spontaneous abortion (RSA), infertility and so on.

Progesterone is the hormone responsible for the secretory changes in the endometrium, it is expected that high progesterone levels in responsible to the hyperechogenic endometrium. Investigations have shown a relationship between the serum progesterone level and secretory changes in the endometrium in controlled ovarian hyperstimulation $(\mathrm{COH})$ cycles $^{[73]}$. Some study suggested that a premature secretory endometrial pattern is caused by the advanced rise of $\mathrm{P}^{[74]}$, and this premature conversion is bad for pregnancy ${ }^{[75]}$. Moreover, another team ${ }^{[76]}$ found that ovarian stimulation would increase PRB expression and lead to the proliferative endometrium persistence. Therefore, delayed endometrial maturation may not be synchronized with embryonic developmental stages. Our study did not analyze the correlation between progesterone levels and endometrial patterns, but in either situation(earlier or later), endometrial development does not match embryo, consequently lead to the increasing EP rate. But there was no difference in frozen embryo cycles, this may be related to the less frequently early $P$ rise in the frozen embryo period. Different unknown mechanisms produced hyperechogenicity, although the exact mechanism is unknown, it is believed that other hormones, such as androgens and exogenous gonadotropins, cause premature echogenicity of the endometrium by direct effects on the endometrium ${ }^{[77,78]}$. Further studies should be done to explore these mechanisms.

Clinically, combining endometrial and embryo information are often considered together. However, in the present study, a suggestion of combining EMT and embryo stage to predict ectopic pregnancy could not be made since the AUC of combining the two factors was as low as 0.604 . Many factors known to affect EP such as the sequelae stage of pelvic inflammatory disease and maternal age cannot be cured before ET. In this study, our initial aim was to analyze only the effects of endometrial and embryonic parameters on EP after matching other well-known influencing factors, hoping that EP can be prevented by changing the parameters of endometrium and embryo to some extent in the IVF-ET cycle, in further study, when we use more of the risk parameters such as number of embryos transferred to set up a model, it would be likely to get a better prediction effect.

In addition, it is necessary to note that the correlation between EMT and pattern and embryo stage and pregnancy outcome shown in our study does not imply a causal relationship, we must acknowledge its function more than its mere thickness and pattern. The relationship may also result from some other factors that are responsible for endometrial receptivity (such as blood flow or some other underlying physiological machinery responsible for periodic endometrial changes). Despite a higher pattern $\mathrm{C}$ rate in EP group, we disagree with the idea that embryo cryopreservation and subsequent ET in a frozen cycle. 
We agree with Friedler ${ }^{[79]}$ that endometrial pattern offers important predictive information but should not be used as an absolute predictor of conception. We believe that such patients should be adequately counseled and given the most adaptive advice, routine endometrial peristaltic wave examination in patients with endometrium pattern $\mathrm{C}$ or thickness less than $9.35 \mathrm{~mm}$ on transformation day may be of certain significance.

This study has some limitation, the most important of which is that it is retrospective in nature, moreover, lifestyle factors such as alcohol consumption, smoking, risky sexual behaviors and some other factors which were previously reported to be associated with higher EP risks ${ }^{[80]}$ were not evaluated. However, we believe the results are of interest because similar but not the same studies have published with debate results, there is no consensus about the optimal cutoff value of EMT and the prediction or prevention strategy of EP after ART. A well-designed and powered randomized clinical trial will be needed to achieve these ends.

\section{Conclusions}

A thin EMT on the day of endometrial transformation and cleavage embryo transferring were independent risk factors for ectopic pregnancy after ART. Combined endometrial and embryonic features were not sufficient variables to predict the ectopic pregnancy during IVF-ET treatment cycles. Efforts to increase the EMT and prolong embryo culture to blastocyst stage may further reduce ectopic pregnancy risk after ART. Endometrial peristaltic wave examination and corresponding treatment before embryo transfer in patients with risk factors may help preventing EP.

\section{List Of Abbreviations}

EP ectopic pregnancy; IUP intrauterine pregnancy; $B M /$ body mass index; $A R T$ assisted reproductive technology; IVF-ET in vitro fertilization-embryo transfer; EMT endometrial thickness

\section{Declarations}

\section{Ethics approval and consent to participate}

The study was approved by Ethics Committee of Xiangya Hospital, Central South University.

\section{Consent for publication}

Not applicable.

\section{Availability of data and materials}

The datasets used and/or analysed during the current study are available from the corresponding author on reasonable request. 


\section{Competing interests}

The authors declare that they have no competing interests.

\section{Funding}

This study was supported by the National Key Research and Developmental Program of China(2018YFC1004800) and the National Natural Science Foundation of China (grant no. 8187061497).

\section{Authors' contribution}

Y.Z. analyzed and interpreted all the patients' data, and was the major contributor in writing the manuscript. All authors participated in data analysis and results discussion, read and approved the final manuscript.

\section{Acknowledgement}

The authors thank everyone in the department of Reproductive Medicine in the Xiangya Hospital of Centre South University for their scientific advice and friendly help.

\section{References}

1. ACOG Practice Bulletin No. 191 Summary: Tubal Ectopic Pregnancy [J],Obstetrics and gynecology,131 (2018) 409-411. 10.1097/aog.0000000000002499.

2. K. Khan, D. Wojdyla, L. Say, et al., WHO analysis of causes of maternal death: a systematic review [J],Lancet (London, England),367 (2006) 1066-1074. 10.1016/s0140-6736(06)68397-9.

3. J. Shaw, S. Dey, H. Critchley, et al., Current knowledge of the aetiology of human tubal ectopic pregnancy [J],Human reproduction update,16 (2010) 432-444. 10.1093/humupd/dmp057.

4. C. Li, C. Meng, W. Zhao, et al., Risk factors for ectopic pregnancy in women with planned pregnancy: a case-control study [J],European journal of obstetrics, gynecology, and reproductive biology,181 (2014) 176-182. 10.1016/j.ejogrb.2014.07.049.

5. M. Kazandi, V. Turan, Ectopic pregnancy; risk factors and comparison of intervention success rates in tubal ectopic pregnancy [J],Clinical and experimental obstetrics \& gynecology,38 (2011) 67-70.

6. V. Akande, C. Turner, P. Horner, et al., Impact of Chlamydia trachomatis in the reproductive setting: British Fertility Society Guidelines for practice [J],Human fertility (Cambridge, England), 13 (2010) 115-125. 10.3109/14647273.2010.513893.

7. M. Gradison, Pelvic inflammatory disease [J],American family physician,85 (2012) 791-796.

8. C. Mitchell, M. Prabhu, Pelvic inflammatory disease: current concepts in pathogenesis, diagnosis and treatment [J],Infectious disease clinics of North America,27 (2013) 793-809. 10.1016/j.idc.2013.08.004 
9. S. Parashi, S. Moukhah, M. Ashrafi, Main risk factors for ectopic pregnancy: a case-control study in a sample of Iranian women [J],International journal of fertility \& sterility,8 (2014) 147-154.

10. M. Patil, Ectopic pregnancy after infertility treatment [J],Journal of human reproductive sciences, 5 (2012) 154-165. 10.4103/0974-1208.101011.

11. P. Yong, S. Matwani, C. Brace, et al., Endometriosis and Ectopic Pregnancy: A Meta-analysis [J],Journal of minimally invasive gynecology,27 (2020) 352-361.e352. 10.1016/j.jmig.2019.09.778.

12. M. Hjordt Hansen, T. Dalsgaard, D. Hartwell, et al., Reproductive prognosis in endometriosis. A national cohort study [J],Acta obstetricia et gynecologica Scandinavica,93 (2014) 483-489. 10.1111/aogs.12373.

13. P. Steptoe, R. Edwards, Reimplantation of a human embryo with subsequent tubal pregnancy [J],Lancet (London, England),1 (1976) 880-882. 10.1016/s0140-6736(76)92096-1.

14. P. Steptoe, R. Edwards, Birth after the reimplantation of a human embryo [J],Lancet (London, England),2 (1978) 366. 10.1016/s0140-6736(78)92957-4.

15. D. Jurkovic, H. Wilkinson, Diagnosis and management of ectopic pregnancy [J],BMJ (Clinical research ed.),342 (2011) d3397. 10.1136/bmj.d3397.

16. V. Muller, M. Makhmadalieva, I. Kogan, et al., Ectopic pregnancy following in vitro fertilization: metaanalysis and single-center experience during 6 years [J],Gynecological endocrinology : the official journal of the International Society of Gynecological Endocrinology,32 (2016) 69-74. 10.1080/09513590.2016.1232550.

17. C. Li, W. Zhao, Q. Zhu, et al., Risk factors for ectopic pregnancy: a multi-center case-control study [J],BMC pregnancy and childbirth,15 (2015) 187. 10.1186/s12884-015-0613-1.

18. H. Clayton, L. Schieve, H. Peterson, et al., Ectopic pregnancy risk with assisted reproductive technology procedures [J],Obstetrics and gynecology,107 (2006) 595-604. 10.1097/01.Aog.0000196503.78126.62.

19. A. Strandell, J. Thorburn, L. Hamberger, Risk factors for ectopic pregnancy in assisted reproduction [J],Fertility and sterility,71 (1999) 282-286. 10.1016/s0015-0282(98)00441-5.

20. E. Pyrgiotis, K. Sultan, G. Neal, et al., Ectopic pregnancies after in vitro fertilization and embryo transfer [J],Journal of assisted reproduction and genetics, 11 (1994) 79-84. 10.1007/bf02215992.

21. M. Malak, T. Tawfeeq, H. Holzer, et al., Risk factors for ectopic pregnancy after in vitro fertilization treatment [J],Journal of obstetrics and gynaecology Canada : JOGC $=$ Journal d'obstetrique et gynecologie du Canada : JOGC,33 (2011) 617-619. 10.1016/s1701-2163(16)34910-6.

22. B. Refaat, E. Dalton, W. Ledger, Ectopic pregnancy secondary to in vitro fertilisation-embryo transfer: pathogenic mechanisms and management strategies [J],Reproductive biology and endocrinology : RB\&E,13 (2015) 30. 10.1186/s12958-015-0025-0.

23. B. Huang, D. Hu, K. Qian, et al., Is frozen embryo transfer cycle associated with a significantly lower incidence of ectopic pregnancy? An analysis of more than 30,000 cycles [J],Fertility and sterility, 102 (2014) 1345-1349. 10.1016/j.fertnstert.2014.07.1245. 
24. B. Shapiro, S. Daneshmand, L. De Leon, et al., Frozen-thawed embryo transfer is associated with a significantly reduced incidence of ectopic pregnancy [J],Fertility and sterility,98 (2012) 1490-1494. 10.1016/j.fertnstert.2012.07.1136.

25. B. Shapiro, S. Daneshmand, F. Garner, et al., Evidence of impaired endometrial receptivity after ovarian stimulation for in vitro fertilization: a prospective randomized trial comparing fresh and frozen-thawed embryo transfer in normal responders [J],Fertility and sterility,96 (2011) 344-348. 10.1016/j.fertnstert.2011.05.050.

26. O. Ishihara, A. Kuwahara, H. Saitoh, Frozen-thawed blastocyst transfer reduces ectopic pregnancy risk: an analysis of single embryo transfer cycles in Japan [J],Fertility and sterility,95 (2011) 19661969. 10.1016/j.fertnstert.2011.02.015.

27. N. Polyzos, P. Devroey, Significantly lower ectopic pregnancy rates after frozen embryo transfer: implications toward segmentation of in vitro fertilization treatment [J],Fertility and sterility,98 (2012) 1419-1420. 10.1016/j.fertnstert.2012.08.044.

28. L. Londra, C. Moreau, D. Strobino, et al., Ectopic pregnancy after in vitro fertilization: differences between fresh and frozen-thawed cycles [J],Fertility and sterility,104 (2015) 110-118. 10.1016/j.fertnstert.2015.04.009.

29. Z. Bu, Y. Xiong, K. Wang, et al., Risk factors for ectopic pregnancy in assisted reproductive technology: a 6-year, single-center study [J],Fertility and sterility,106 (2016) 90-94. 10.1016/j.fertnstert.2016.02.035.

30. A. Milki, S. Jun, Ectopic pregnancy rates with day 3 versus day 5 embryo transfer: a retrospective analysis [J],BMC pregnancy and childbirth,3 (2003) 7. 10.1186/1471-2393-3-7.

31. D. Keegan, S. Morelli, N. Noyes, et al., Low ectopic pregnancy rates after in vitro fertilization: do practice habits matter? [J],Fertility and sterility,88 (2007) 734-736. 10.1016/j.fertnstert.2006.11.169.

32. E. Rosman, D. Keegan, L. Krey, et al., Ectopic pregnancy rates after in vitro fertilization: a look at the donor egg population [J],Fertility and sterility,92 (2009) 1791-1793. 10.1016/j.fertnstert.2009.05.041.

33. L. Marion, G. Meeks, Ectopic pregnancy: History, incidence, epidemiology, and risk factors [J],Clinical obstetrics and gynecology,55 (2012) 376-386. 10.1097/GRF.0b013e3182516d7b.

34. J. Cai, L. Liu, X. Jiang, et al., Low body mass index is associated with ectopic pregnancy following assisted reproductive techniques: a retrospective study [J],BJOG : an international journal of obstetrics and gynaecology,(2020). 10.1111/1471-0528.16378.

35. K. Perkins, S. Boulet, D. Kissin, et al., Risk of ectopic pregnancy associated with assisted reproductive technology in the United States, 2001-2011 [J],Obstetrics and gynecology,125 (2015) 70-78. 10.1097/aog.0000000000000584.

36. W. Ankum, B. Mol, F. Van der Veen, et al., Risk factors for ectopic pregnancy: a meta-analysis [J],Fertility and sterility,65 (1996) 1093-1099.

37. M. Weigert, D. Gruber, E. Pernicka, et al., Previous tubal ectopic pregnancy raises the incidence of repeated ectopic pregnancies in in vitro fertilization-embryo transfer patients [J],Journal of assisted reproduction and genetics,26 (2009) 13-17. 10.1007/s10815-008-9278-2. 
38. S. Jwa, S. Seto, M. Takamura, et al., Ovarian stimulation increases the risk of ectopic pregnancy for fresh embryo transfers: an analysis of 68,851 clinical pregnancies from the Japanese Assisted Reproductive Technology registry [J],Fertility and sterility, 114 (2020) 1198-1206. 10.1016/j.fertnstert.2020.06.032.

39. C. Fang, R. Huang, L. Wei, et al., Frozen-thawed day 5 blastocyst transfer is associated with a lower risk of ectopic pregnancy than day 3 transfer and fresh transfer [J],Fertility and sterility,103 (2015) 655-661.e653. 10.1016/j.fertnstert.2014.11.023.

40. W. Xing, J. Ou, L. Cai, Thawed embryo transfer and ectopic pregnancy: a meta-analysis [J],Archives of gynecology and obstetrics,297 (2018) 1345-1352. 10.1007/s00404-018-4724-6.

41. M. Bredella, V. Feldstein, R. Filly, et al., Measurement of endometrial thickness at US in multicenter drug trials: value of central quality assurance reading [J],Radiology,217 (2000) 516-520. 10.1148/radiology.217.2.ro0nv34516.

42. J. Zhao, Q. Zhang, Y. Wang, et al., Endometrial pattern, thickness and growth in predicting pregnancy outcome following 3319 IVF cycle [J],Reproductive biomedicine online,29 (2014) 291-298. 10.1016/j.rbmo.2014.05.011.

43. R. Li, Y. Dong, Y. Guo, et al., Comparative study of pregnancy outcomes between day 3 embryo transfer and day 5 blastocyst transfer in patients with progesterone elevation [J],The Journal of international medical research,41 (2013) 1318-1325. 10.1177/0300060513489480.

44. T. Du, H. Chen, R. Fu, et al., Comparison of ectopic pregnancy risk among transfers of embryos vitrified on day 3, day 5, and day 6 [J],Fertility and sterility, 108 (2017) 108-116.e101. 10.1016/j.fertnstert.2017.05.027.

45. B. Zhang, L. Cui, R. Tang, et al., Reduced Ectopic Pregnancy Rate on Day 5 Embryo Transfer Compared with Day 3: A Meta-Analysis [J],PloS one,12 (2017) e0169837. 10.1371/journal.pone.0169837.

46. D. Gardner, P. Vella, M. Lane, et al., Culture and transfer of human blastocysts increases implantation rates and reduces the need for multiple embryo transfers [J],Fertility and sterility,69 (1998) 84-88. 10.1016/s0015-0282(97)00438-x.

47. L. Smith, S. Oskowitz, L. Dodge, et al., Risk of ectopic pregnancy following day-5 embryo transfer compared with day-3 transfer [J],Reproductive biomedicine online,27 (2013) 407-413. 10.1016/j.rbmo.2013.06.015.

48. L. Cheng, P. Lin, F. Huang, et al., Ectopic pregnancy following in vitro fertilization with embryo transfer: A single-center experience during 15 years [J],Taiwanese journal of obstetrics \& gynecology,54 (2015) 541-545. 10.1016/j.tjog.2015.08.004.

49. E. Wang, A. Kathiresan, C. Bresee, et al., Abnormal implantation after fresh and frozen in vitro fertilization cycles [J],Fertility and sterility,107 (2017) 1153-1158. 10.1016/j.fertnstert.2017.03.012.

50. S. Wang, H. Sun, Blastocyst transfer ameliorates live birth rate compared with cleavage-stage embryos transfer in fresh in vitro fertilization or intracytoplasmic sperm injection cycles: reviews and meta-analysis [J],Yonsei medical journal,55 (2014) 815-825. 10.3349/ymj.2014.55.3.815. 
51. H. Chang, C. Suh, Ectopic pregnancy after assisted reproductive technology: what are the risk factors? [J],Current opinion in obstetrics \& gynecology,22 (2010) 202-207. 10.1097/GC0.0b013e32833848fd.

52. S. Arya, S. Kupesic Plavsic, Preimplantation 3D ultrasound: current uses and challenges [J],Journal of perinatal medicine,45 (2017) 745-758. 10.1515/jpm-2016-0361.

53. G. Basir, W. O, W. So, et al., Evaluation of cycle-to-cycle variation of endometrial responsiveness using transvaginal sonography in women undergoing assisted reproduction [J],Ultrasound in obstetrics \& gynecology : the official journal of the International Society of Ultrasound in Obstetrics and Gynecology, 19 (2002) 484-489. 10.1046/j.1469-0705.2002.00685.x.

54. R. Dickey, T. Olar, D. Curole, et al., Endometrial pattern and thickness associated with pregnancy outcome after assisted reproduction technologies [J],Human reproduction (Oxford, England),7 (1992) 418-421. 10.1093/oxfordjournals.humrep.a137661.

55. R. Fanchin, Assessing uterine receptivity in 2001: ultrasonographic glances at the new millennium [J],Annals of the New York Academy of Sciences,943 (2001) 185-202. 10.1111/j.17496632.2001.tb03802.x.

56. L. Wang, J. Qiao, R. Li, et al., Role of endometrial blood flow assessment with color Doppler energy in predicting pregnancy outcome of IVF-ET cycles [J],Reproductive biology and endocrinology : RB\&E,8 (2010) 122. 10.1186/1477-7827-8-122.

57. Y. Yuval, S. Lipitz, J. Dor, et al., The relationships between endometrial thickness, and blood flow and pregnancy rates in in-vitro fertilization [J],Human reproduction (Oxford, England),14 (1999) 10671071. 10.1093/humrep/14.4.1067.

58. G. McWilliams, J. Frattarelli, Changes in measured endometrial thickness predict in vitro fertilization success [J],Fertility and sterility,88 (2007) 74-81. 10.1016/j.fertnstert.2006.11.089.

59. W. Amir, B. Micha, H. Ariel, et al., Predicting factors for endometrial thickness during treatment with assisted reproductive technology [J],Fertility and sterility,87 (2007) 799-804. 10.1016/j.fertnstert.2006.11.002.

60. K. Richter, K. Bugge, J. Bromer, et al., Relationship between endometrial thickness and embryo implantation, based on 1,294 cycles of in vitro fertilization with transfer of two blastocyst-stage embryos [J],Fertility and sterility,87 (2007) 53-59. 10.1016/j.fertnstert.2006.05.064.

61. X. Zhang, C. Chen, E. Confino, et al., Increased endometrial thickness is associated with improved treatment outcome for selected patients undergoing in vitro fertilization-embryo transfer [J],Fertility and sterility,83 (2005) 336-340. 10.1016/j.fertnstert.2004.09.020.

62. U. Zenke, R. Chetkowski, Transfer and uterine factors are the major recipient-related determinants of success with donor eggs [J],Fertility and sterility,82 (2004) 850-856.

10.1016/j.fertnstert.2004.03.057.

63. P. Kovacs, S. Matyas, K. Boda, et al., The effect of endometrial thickness on IVF/ICSI outcome [J],Human reproduction (Oxford, England),18 (2003) 2337-2341. 10.1093/humrep/deg461. 
64. X. Liu, P. Qu, H. Bai, et al., Endometrial thickness as a predictor of ectopic pregnancy in 1125 in vitro fertilization-embryo transfer cycles: a matched case-control study [J],Archives of gynecology and obstetrics,300 (2019) 1797-1803. 10.1007/s00404-019-05353-z.

65. H. Liu, J. Zhang, B. Wang, et al., Effect of endometrial thickness on ectopic pregnancy in frozen embryo transfer cycles: an analysis including 17,244 pregnancy cycles [J],Fertility and sterility, 113 (2020) 131-139. 10.1016/j.fertnstert.2019.09.003.

66. M. Kabir-Salmani, C. Murphy, A. Hosseini, et al., Ultrastructural Modifications of Human Endometrium during the Window of Implantation [J],Int J Fertil Steril,2 (2007).

67. L. Rombauts, C. Motteram, E. Berkowitz, et al., Risk of placenta praevia is linked to endometrial thickness in a retrospective cohort study of 4537 singleton assisted reproduction technology births [J],Human reproduction (Oxford, England),29 (2014) 2787-2793. 10.1093/humrep/deu240.

68. F. Sharara, J. Lim, H. McClamrock, Endometrial pattern on the day of oocyte retrieval is more predictive of implantation success than the pattern or thickness on the day of hCG administration [J],Journal of assisted reproduction and genetics,16 (1999) 523-528. 10.1023/a:1020545120256.

69. A. Vera J, B. Argüello, A. Crisosto C, [Predictive value of endometrial pattern and thickness in the result of in vitro fertilization and embryo transfer] [J],Revista chilena de obstetricia y ginecologia, 60 (1995) 195-198.

70. R. Fanchin, C. Righini, J. Ayoubi, et al., New look at endometrial echogenicity: objective computerassisted measurements predict endometrial receptivity in in vitro fertilization-embryo transfer [J],Fertility and sterility,74 (2000) 274-281. 10.1016/s0015-0282(00)00643-9.

71. A. Fleischer, G. Kalemeris, S. Entman, Sonographic depiction of the endometrium during normal cycles [J],Ultrasound in medicine \& biology,12 (1986) 271-277. 10.1016/0301-5629(86)90336-4.

72. L. Grunfeld, B. Walker, P. Bergh, et al., High-resolution endovaginal ultrasonography of the endometrium: a noninvasive test for endometrial adequacy [J],Obstetrics and gynecology,78 (1991) 200-204.

73. R. Chetkowski, R. Kiltz, W. Salyer, In premature luteinization, progesterone induces secretory transformation of the endometrium without impairment of embryo viability [J],Fertility and sterility, 68 (1997) 292-297. 10.1016/s0015-0282(97)81518-x.

74. R. Fanchin, C. Righini, F. Olivennes, et al., Computerized assessment of endometrial echogenicity: clues to the endometrial effects of premature progesterone elevation [J],Fertility and sterility,71 (1999) 174-181. 10.1016/s0015-0282(98)00410-5.

75. J. Zhao, Q. Zhang, Y. Li, The effect of endometrial thickness and pattern measured by ultrasonography on pregnancy outcomes during IVF-ET cycles [J],Reproductive biology and endocrinology : RB\&E,10 (2012) 100. 10.1186/1477-7827-10-100.

76. L. Detti, G. Saed, N. Fletcher, et al., Endometrial morphology and modulation of hormone receptors during ovarian stimulation for assisted reproductive technology cycles [J],Fertility and sterility, 95 (2011) 1037-1041. 10.1016/j.fertnstert.2010.12.025. 
77. A. Corbacioglu, B. Baysal, Effects of steroid hormone levels on the ultrasound appearance of the preovulatory endometrium in controlled ovarian hyperstimulation cycles [J],International journal of fertility \& sterility,5 (2012) 203-206.

78. L. Detti, F.D. Yelian, M.L. Kruger, et al., Endometrial thickness is related to miscarriage rate, but not to the estradiol concentration, in cycles down-regulated with gonadotropin-releasing hormone antagonist [J],Fertility \& Sterility,89 (2008) 998-1001.

79. S. Friedler, J. Schenker, A. Herman, et al., The role of ultrasonography in the evaluation of endometrial receptivity following assisted reproductive treatments: a critical review [J],Human reproduction update,2 (1996) 323-335. 10.1093/humupd/2.4.323.

80. A. Gaskins, S. Missmer, J. Rich-Edwards, et al., Demographic, lifestyle, and reproductive risk factors for ectopic pregnancy [J],Fertility and sterility,110 (2018) 1328-1337.

10.1016/j.fertnstert.2018.08.022.

\section{Figures}

IVF-ET/ICSI cycles between January 2014 and April 2020 $(n=21704)$

include: good physical and mental ; positive hCG test 12 days after embryo transfer
hCG positive ( $n=11699)$

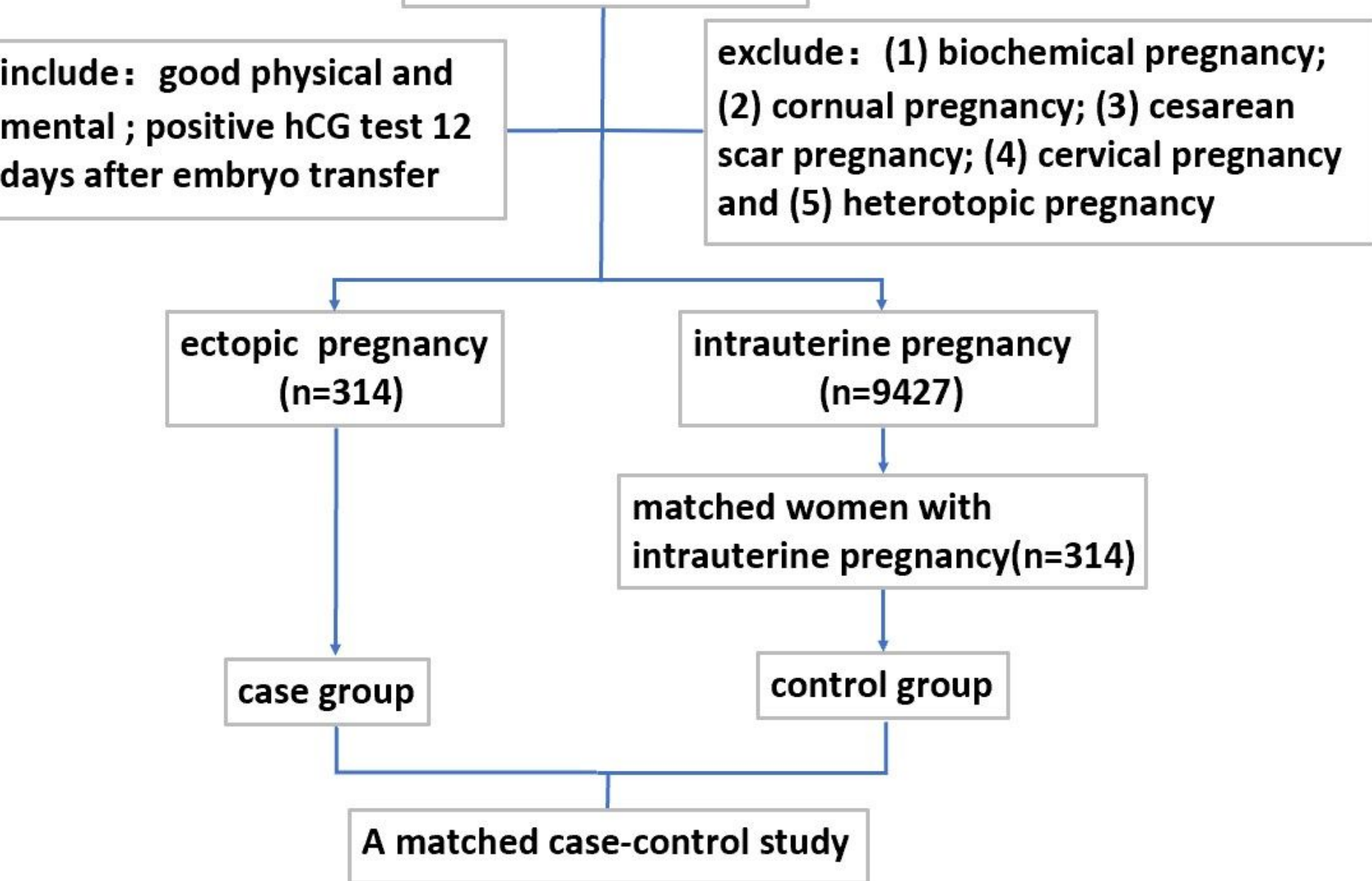


Figure 1

Patient selection flowchart. IVF-ET in vitro fertilization-embryo transfer, ICSI intracytoplasmic sperm injection, hCG human chorionic gonadotropin

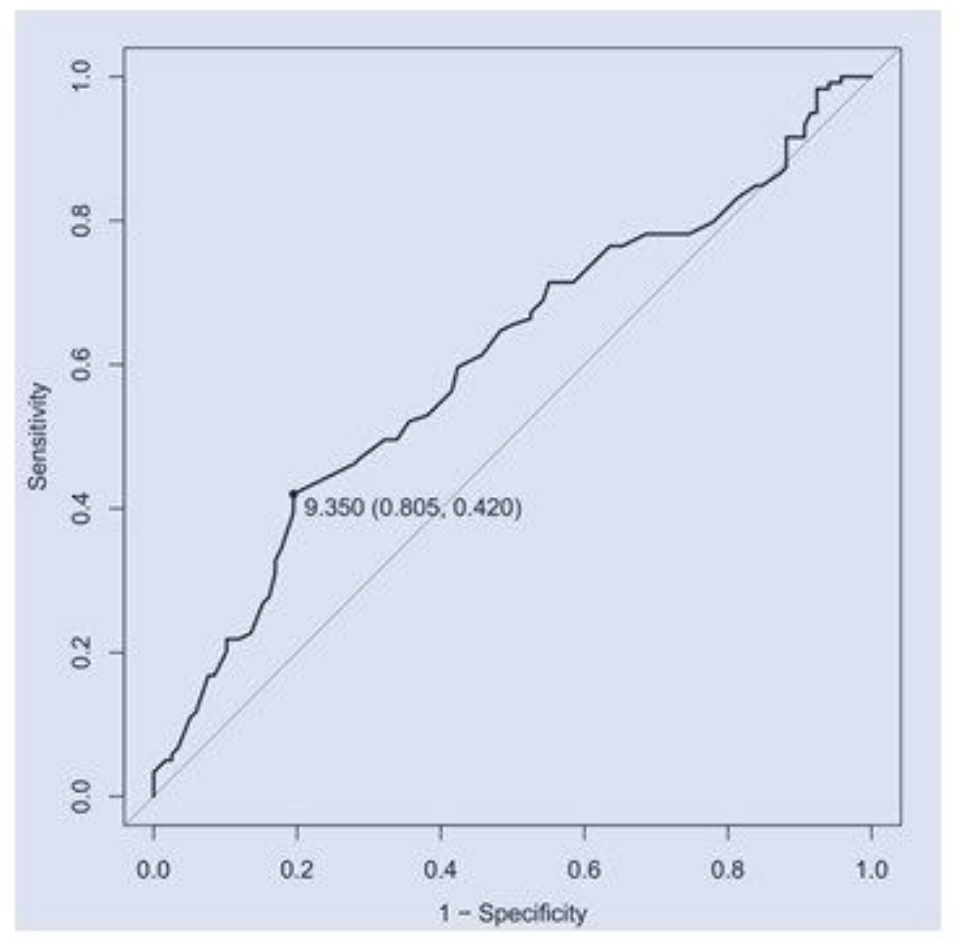

\section{Figure 2}

The cutoff value of the EMT on trigger day in fresh cycles. The cutoff value was $9.35 \mathrm{~mm}$.

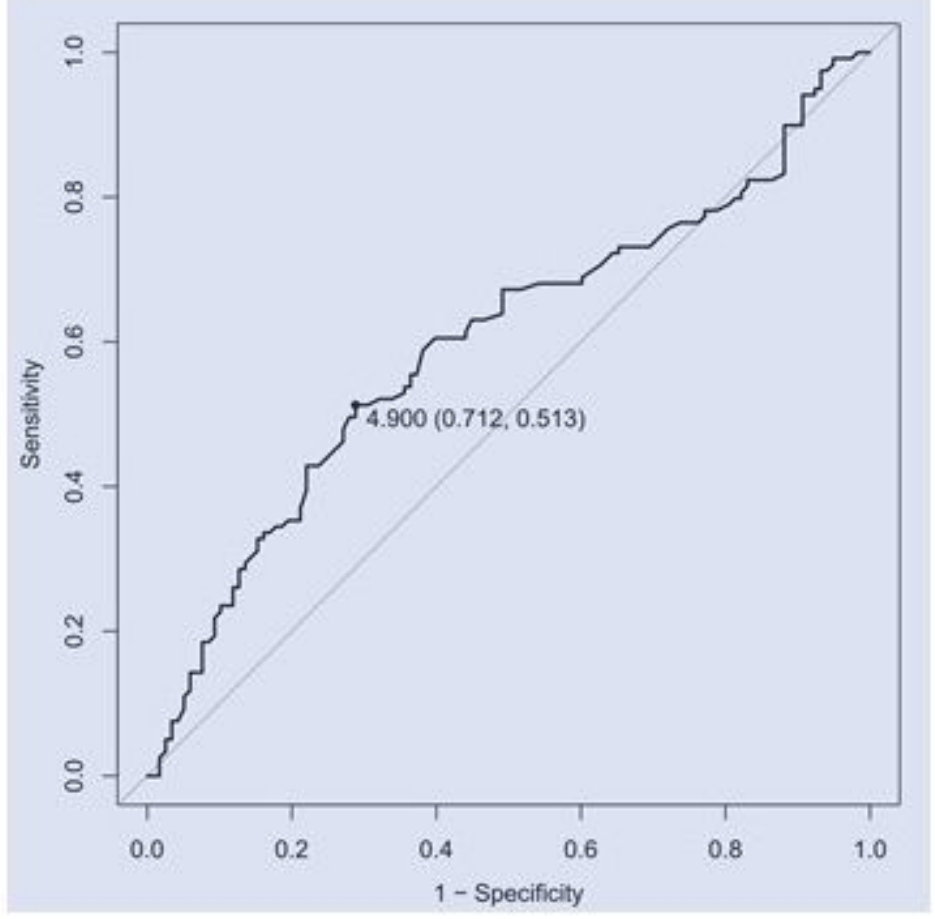

Figure 3 
The cutoff value of the endometrial growth amplitude in fresh cycles. The cutoff value was $4.9 \mathrm{~mm}$.

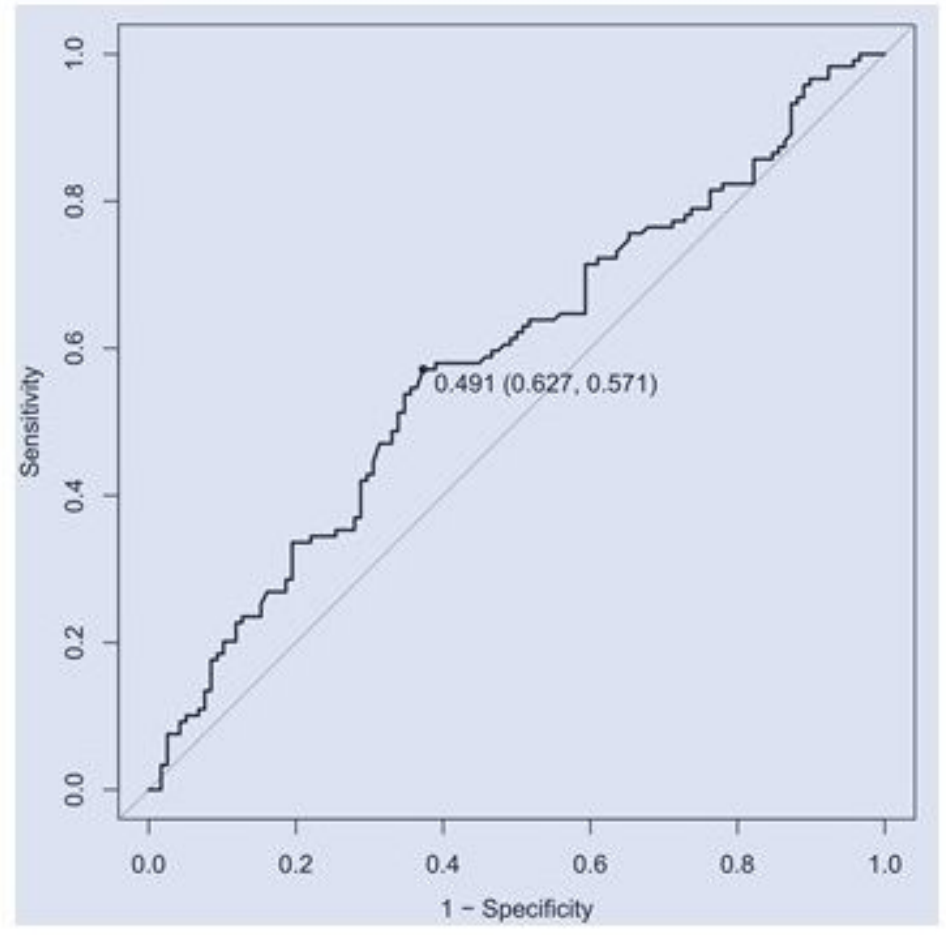

\section{Figure 4}

The cutoff value of the endometrial growth rate in fresh cycles. The cutoff value was $0.491 \mathrm{~mm} /$ day.

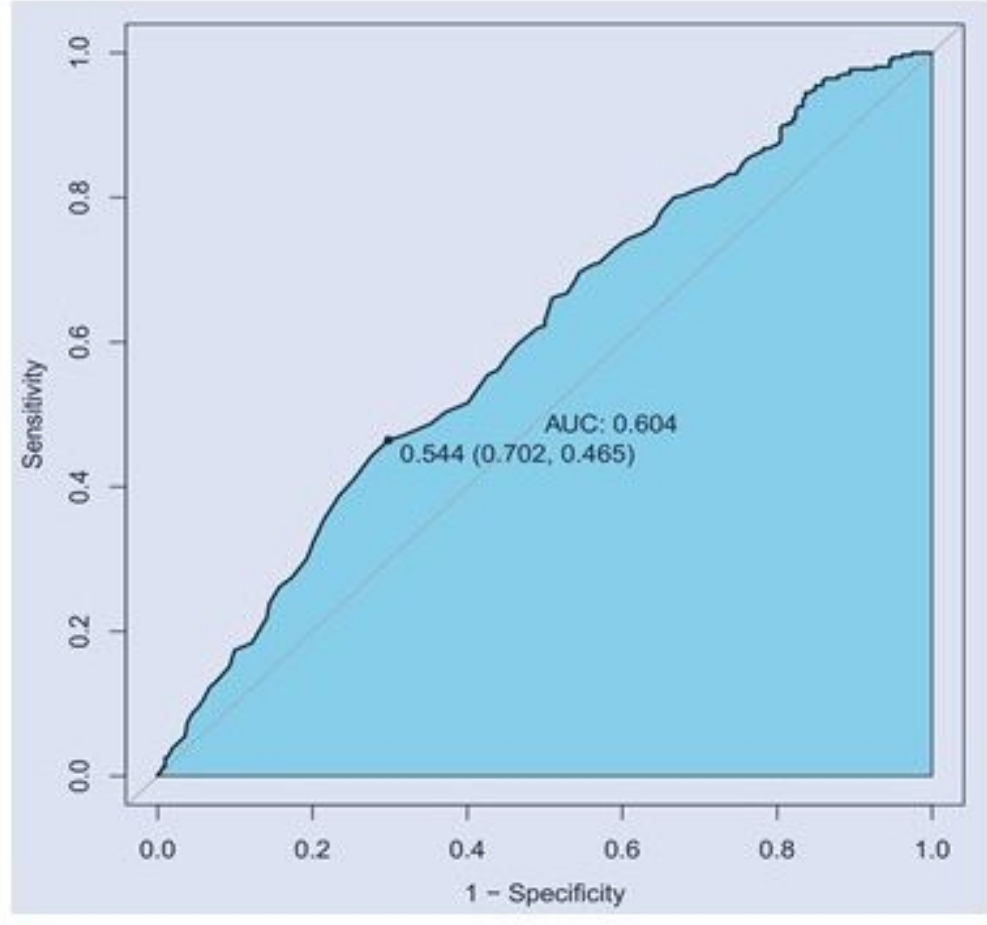

\section{Figure 5}

Receiver operator characteristic curve of EMT on transformation day and stage of embryos transferred. The area under the curve was 0.604 . Diagonal segments are produced by ties. 


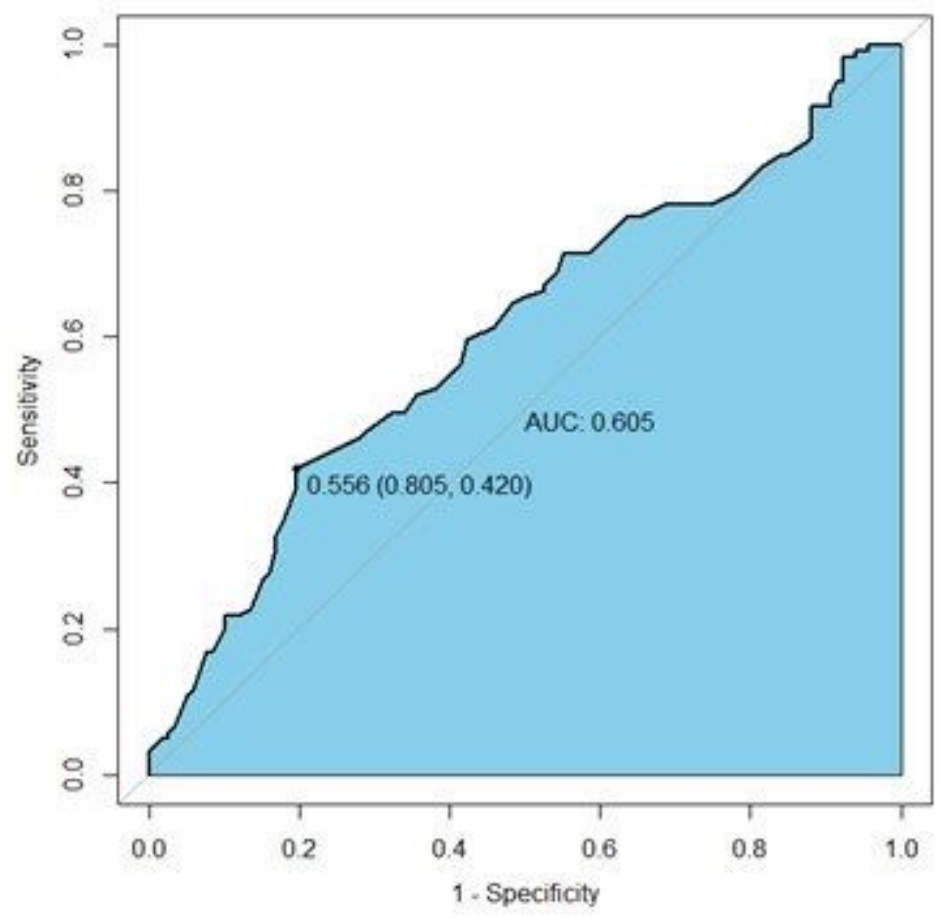

Figure 6

Receiver operator characteristic curve of EMT on trigger day for fresh cycles. The area under the curve was 0.605 . Diagonal segments are produced by ties. 